\title{
Rectilinear Scan
}

National Cancer Institute

\section{Source}

National Cancer Institute. Rectilinear Scan. NCI Thesaurus. Code C94969.

A very simplified scan, now of historical interest, in which a radiation detector is

systematically positioned over the subject, viewing only a very small region at a time. 